\title{
Management of peri- ocular skin tumours by laissez-faire technique: analysis of functional and cosmetic results
}

Abstract

Aim The role of wound healing by secondary intention in the treatment of periocular skin tumours is not well established. The object of this retrospective analysis was to evaluate the functional and cosmetic outcome of patients treated by the Laissezfaire technique in situations where primary closure would not have been possible.

Methods Skin defects following excision of lid and peri-ocular tumours in 24 Caucasian patients were allowed to heal by granulation. The locations included lower eyelid $(n=10)$, upper lid $(n=6)$, medial canthus $(n=5)$, nasojugal fold $(n=2)$, lateral canthus $(n=1)$ and brow $(n=1)$. Four patients had lid margin involvement. The size of the initial defect, time taken to heal, discomfort during healing, the functional and cosmetic results-both from the surgeon and patient perspective, complications, secondary intervention if any and patient satisfaction were studied.

Department of Ophthalmology Royal Glamorgan General Hospital

Ynynsmaerdy

Llantrisant CF72 8XR

Wales

Correspondence:

J Shankar

24 Frankby Road

West Kirby CH48 6EE

Wirral, UK

Tel: 01516258470

E-mail: jai.shankar@

tesco.net

Received: 30 March 2001 Accepted in revised form: 20 August 2001
Results A good functional and cosmetic result was obtained in 23 of the 25 lesions (92\%). Of these 23 patients, two patients had slightly hypertrophied scars, which responded well to massage and two patients had some degree of ectropion. Of the two patients who did not have a good cosmetic result, only one needed secondary intervention. One had an exuberant granulation tissue, which responded to topical steroids and massage, but left behind a distorted lateral canthus.

Conclusion Healing by secondary intention of large defects following excision of periocular tumours is an effective alternative to primary or staged reconstruction in selected cases.
J Shankar, RG Nair and SC Sullivan

Eye (2002) 16, 50-53. DOI: 10.1038/

sj/EYE/6700057

Keywords: eyelid; tumours; laissez-faire; wound healing; secondary healing; results

\section{Introduction}

Healing by secondary intention has been a time-honoured method of wound management in general and plastic surgery but its application in peri-ocular reconstruction has been less popular. The fear of sub-optimal cosmetic and functional outcome in lesions involving the lid has tempered the enthusiasm for this technique. The objective of this study was to examine the role of secondary intention in the healing following excision of peri-ocular skin tumours in cases that would have normally required extensive flaps or skin grafts to close the defect.

\section{Methods}

Twenty-four consecutive patients underwent excision of peri-ocular skin tumours without primary reconstruction between July 1996 and February 1999. These 24 patients represented 25 lesions, one patient having two lesions. Incisional biopsies were performed in all cases prior to definitive surgery. Excisions were performed under local anaesthesia. Lesions were excised including a 3-mm margin around the clinically visible extent of the tumour. Four patients had a full thickness excision of the lid. All excised tissue was mapped and subjected to histopathological examination to confirm the diagnosis and to assess tumour clearance. The peri-ocular defect was allowed to granulate. The decision 
to allow the wound to heal by secondary intention was made, with patient consent after all treatment options had been discussed, if it was felt that direct primary closure was not possible. None of the patients underwent any form of primary or staged reconstruction, either partial or complete.

Immediately postoperatively, the wounds were dressed with paraffin gauze if the lid margin was involved or with Kaltostat ${ }^{\mathrm{R}}$ (Calcium-sodium alginate) if the lid margin was not involved. The first dressing was removed at one week in the clinic. If the granulation tissue did not demonstrate significant serous exudation, no dressing was reapplied and the patient was instructed to keep the area clean and dry. If serous exudation was significant, paraffin gauze or Kaltostat dressing was reapplied for a further week. No topical or systemic antibiotics were administered routinely. No form of adjuvant therapy in the form of cryotherapy or radiotherapy was instituted.

Subsequently, patients were advised to massage the wound if it showed evidence of hypertrophic scarring. The wounds were photographed at each postoperative visit and evaluated for the size of the defect, cosmetic and functional result, complications and secondary intervention, if any.

An independent observer (JS) who was not involved in the management of the case assessed the cosmetic and functional result. They were placed in five categories-excellent, very good, good, acceptable and poor. Patients were asked to fill out a questionnaire based on the visual analogue scale to assess three parameters: (i) discomfort during healing; (ii) satisfaction with the number of postoperative hospital visits; and (iii) cosmetic result from the patient's perspective.

\section{Results}

The cohort consisted of 15 females and nine males. Mean age was 66.04 years (32-94 years). Table 1 shows

Table 1 Location of peri-ocular skin lesion

\begin{tabular}{lccc}
\hline & $\begin{array}{c}\text { With no lid } \\
\text { margin } \\
\text { involvement }\end{array}$ & $\begin{array}{c}\text { With lid } \\
\text { margin } \\
\text { involvement }\end{array}$ & Total \\
\hline Lower lid & 7 & 3 & 10 \\
Upper lid & 6 & 0 & 6 \\
Medial canthus & 4 & 1 & 5 \\
Naso-jugal fold & 2 & 0 & 2 \\
Lateral canthus & 1 & 0 & 1 \\
Brow & 1 & 0 & 1 \\
Total & 21 & 4 & 25 \\
\hline
\end{tabular}
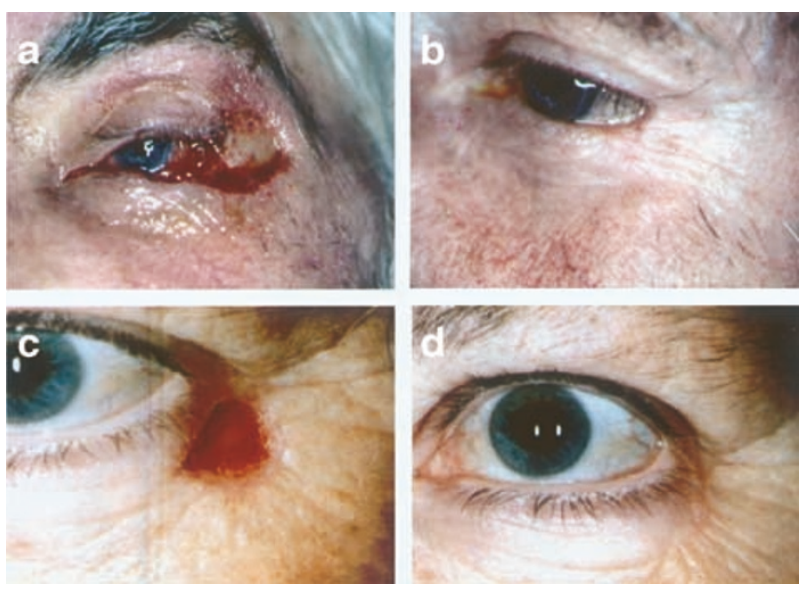

Figure 1 (a) Removal of lateral third of upper lid and lateral half of lower lid. (b) Appearance of the same eye after 5 weeks. (c) An 8-mm defect of the lateral aspect of the lower lid without marginal involvement. (d) Complete healing with an imperceptible scar.

the location of the lesion and lid margin involvement. The lower lid was the most common location.

The size of the initial skin defect ranged from $6 \mathrm{~mm}$ $\times 7 \mathrm{~mm}$ to $15 \mathrm{~mm} \times 32 \mathrm{~mm}$. All wound epithelised within 2-6 weeks.

Histologically, the excision proved to be complete in all but two lesions. These two were tumour-free following second excision. Definitive histology confirmed the preoperative incisional biopsy in all but one equivocal lesion that was subsequently diagnosed as a dermo-lipoma. Of the 25 lesions, 23 were reported as basal cell carcinoma, one as squamous cell carcinoma and one as benign dermo-lipoma. Follow-up ranged from 2 months to 6 months.

A 'good' or 'very good' result was obtained in $92 \%$ of cases $(23 / 25)$ without further intervention (Figure 1) and was independent of patient age. Three patients had cicatricial ectropion but only one was significant enough to require any secondary surgical intervention (Figure 2a and b). One patient had an unsightly exuberant granulation tissue but this responded to topical steroids and massage (Figure $2 \mathrm{c}$ and $\mathrm{d}$ ). This patient also had lateral canthal distortion. Two had slightly hypertrophied scars that responded well to simple massage (Table 2). Wounds were covered by normal appearing epithelium. In those patients treated

Table 2 Complications encountered

\begin{tabular}{lll} 
Scarring + ectropion & 3 cases & $\begin{array}{l}\text { (1 needing secondary } \\
\text { intervention) }\end{array}$ \\
Granulation tissue & 1 case & (no secondary intervention) \\
Hypertrophied scar & 2 cases & (no secondary intervention) \\
\hline
\end{tabular}



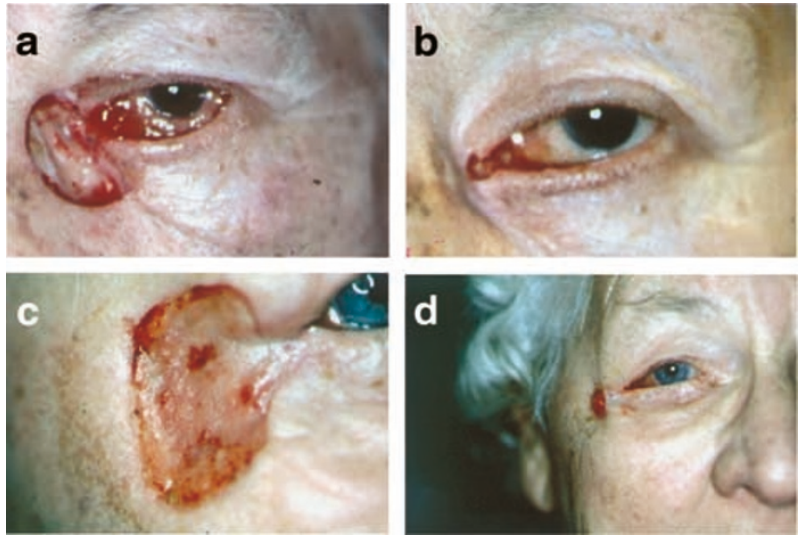

Figure 2 (a) 24-mm medial canthal defect exposing periosteum after lacrimal sac fossa clearance. (b) Healing in 6 weeks with minimal residual ectropion. (c) A 33- $\mathrm{mm} \times 18$-mm defect adjacent to lateral canthus. (d) Healing leaving a granuloma and lateral canthal distortion-resolved following massage with steroid cream.

by full thickness excision of lid, the newly formed lid was of similar consistency to normal tarsus and afforded a stable lid margin. No patient had any exposure keratopathy or ocular irritation. No specific bacterial pathogen was isolated in the patient with the exuberant granulation tissue. Figure 3 shows the results of the patient questionnaire based on a visual analogue scale.

\section{Discussion}

Small peri-ocular skin defects can be closed easily by direct closure aided by lax and redundant tissue, particularly in the elderly. However, larger defects may require reconstruction with a combination of skin mobilisation, skin flaps or free full thickness skin grafts. These techniques are not without cosmetic and functional limitations. ${ }^{1}$ Allowing the wound to heal by secondary intention is a viable alternative. In our series we obtain a 'good' or 'very good' functional and cosmetic result in 23 of the 25 cases (92\%). Only one of the 25 lesions (4\%), necessitated any form of secondary reconstructive procedure. Lowry et $a l^{2}$ presented a series of 59 patients with a satisfactory functional and cosmetic result in $83 \%$ of them. Only two of his patients $(3.4 \%)$ required secondary repair. These results are comparable to our series. Only one among the 11 patients described by $\mathrm{Mehta}^{3}$ needed secondary repair after excision of lesions involving lid margin. He, however, did not describe any involving the lateral canthus or lesions distant from the lid margin. We had one case involving the lateral canthus, which resulted in an excellent cosmetic and functional outcome.

Wohlrab et $a l^{4}$ achieved a satisfactory result in all 12 of

\section{$\square$ visual analogue scale}

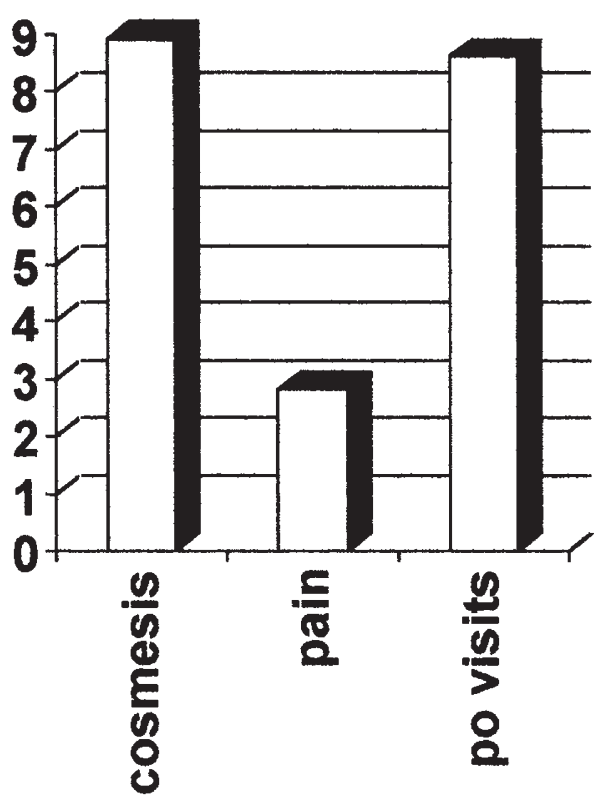

po $=$ post-operative

Figure 3 Results of patient questionnaire.

their cases. Zitelli, ${ }^{5}$ in his monograph on healing by secondary intention described the NEET, NOCH and FAIR areas of the face. The NEET areas-concave surfaces of the nose, eye, ear and temple heal with excellent cosmetic result. Wounds located on the convex surfaces of the nose, oral lips, cheeks and chin, and the helix of the ear (NOCH areas) often heal with noticeable scars. Wounds on the flat surfaces of the forehead, antihelix, eyelid (I) and the remainder of the nose, lips and cheek (FAIR areas) produce intermediate results.

The principal indications for a laissez-faire approach to peri-ocular skin tumours are for medial canthal lesions, particularly after radiotherapy, where the skin may be unsuitable for flaps or free skin grafts. ${ }^{6}$ It may also be useful in patients with primary skin precancerous conditions like xeroderma pigmentosa or in patients with poor general health who may not be fit for general anaesthesia or prolonged surgical procedures including complex skin flaps or skin grafting under local anaesthesia. Secondary reconstruction, if required at a later date, is likely to be a far less extensive procedure.

Laissez-faire may also be superior to primary skin grafting or flaps for tumour surveillance where detection of tumour recurrences beneath or adjacent to 
the reconstructed area may be delayed. The simplicity of wound management for patient or carer associated with this technique is an additional benefit.

The disadvantage of laissez-faire is the prolonged healing time and small risk of a secondary surgical intervention. Finally, the technique may not be suited for large upper lid defects where there may the risk of exposure keratitis during wound healing.

\section{Conclusion}

Healing by secondary intention is an effective, safe and inexpensive alternative to primary or staged skin closure in the management of selected peri-ocular skin tumours. It may be particularly useful in the elderly and infirm.

\section{Acknowledgements}

The authors do not have any proprietary interest.

This paper was awarded the Royal Eye Hospital,
London Prize for the best poster at the Annual Congress of the Royal College of Ophthalmologists held in May 2001 at Birmingham, UK.

\section{References}

1 Hanake E. Treatment of skin tumours of the inner canthal region-reconstructing the medial canthal area: a dilemma between doing too much and an aesthetic outcome. Facial Plast Surg 1997; 13: 101-110.

2 Lowry JC, Bartley GB, Garrity JA. Ophthalmol Plast Reconstr Surg 1997; 13: 174-188.

3 Mehta HK. Spontaneous reformation of the lower lid. $\mathrm{Br} J$ Ophthalmol 1981; 65: 202-208.

4 Wohlrab TM, Rohsbach JM, Thiel HJ. Ger J Ophthalmol 1996; 5: 246-249.

5 Zitelli JA. Secondary intention healing: an alternative to surgical repair. Clin Dermatol 1984; 2: 92-106.

6 Colin JRO. Eye lid reconstruction \& tumour management In: Colin JRO (ed). A Manual of Systematic Eye Lid Surgery. Churchill Livingstone: Edinburgh, 1989. 